\title{
Algoritmos de inteligencia artificial para optimización de procesos en la industria plástica
}

\author{
Pablo Patricio Beltrán Caicedo \\ ppbeltran@uce.edu.ec \\ Universidad Central del Ecuador, Ecuador \\ Luis Felipe Borja \\ lfborja@uce.edu.ec \\ Universidad Central del Ecuador, Ecuador
}

\section{RESUMEN}

La optimización de procesos con objetivos múltiples y restricciones inherentes de diversa índole en la industria plástica, es la motivación principal de esta investigación. Esto debido al alto impacto de disponer una metodología formal alternativa en la búsqueda de soluciones a problemas variados dentro de esta industria. Ante esta problemática buscamos una solución utilizando Inteligencia Artificial (IA) y una de las ramas más estudiadas dentro de la optimización que es la computación evolutiva y sus Algoritmos Genéticos (AG). Estos algoritmos se basan en la selección natural y la genética para evolucionar de una población inicial hacia mejores espacios de búsqueda, esta evolución se realiza mediante operaciones de selección, mutación y crossover (recombinación). Logramos implementar una técnica eficiente en la resolución de un problema de optimización, tomando como caso de uso, encontrar las cantidades óptimas de cada componente junto con la reducción del factor de mantenimiento en la elaboración de un producto terminado de plástico, obteniendo como resultado final la minimización del costo de la manufactura.

Palabras clave: inteligencia artificial; algoritmos genéticos; optimización; industria plástica 


\title{
Artificial intelligence algorithms for the optimization process in the plastics industry
}

\begin{abstract}
The optimization of processes with multiple objectives and inherent restrictions from different natures in the plastics industry, is the main motivation for this research. Due to the high impact of providing a formal alternative methodology in the search for solutions to various problems within this industry. In the light of this problem, A solution was searched using Artificial Intelligence (AI) and one of the most studied branches of optimization, Evolutionary Computing and Genetic Algorithms (AG). These algorithms are based on natural selection and genetics to evolve from an initial population to better search spaces, this evolution is carried out through selection, mutation, and crossover (recombination) operations.

It was implemented an efficient technique solving an optimization problem, as a case study, It was found the optimal amounts of each component and reduced the maintenance factor in the elaboration of a finished plastic product. As a result, it was undeniable the reduction of manufacturing cost.
\end{abstract}

Keywords: artificial intelligence; genetic algorithms; optimization; plastic industry

Artículo recibido: 30 noviembre. 2021 Aceptado para publicación: 29 diciembre 2021

Correspondencia: ppbeltran@uce.edu.ec Conflictos de Interés: Ninguna que declarar 


\section{INTRODUCCIÓN}

La Investigación de Operaciones (IO) ha tenido éxito en la optimización de procesos dentro de la industria, mediante el uso de técnicas de programación lineal, programación entera y programación no lineal (Hillier \& Lieberman, 2013). Las cuales han probado ser efectivas para abordar una amplia variedad de problemas prácticos dentro de la industria como: Planeación Logística, Mejora en el Desempeño de Ventas y Manufactura, Optimización del uso y Movimiento de Materias Primas, Reducción de Tiempo de Manufactura y Niveles de Inventario (Hillier \& Lieberman, 2013). Este enfoque sin embargo en algunos casos resulta ser insuficiente en el manejo de problemas en procesos industriales más complejos, esta complejidad se deriva de la incertidumbre y la satisfacción de múltiples objetivos de manera simultanea en donde es necesario usar métodos metaheurísticos para llegar a una solución más cercana a la realidad (Velez \& Montoya, 2007).

La IA ha sido una herramienta de amplio uso y de extendido alcance en los diferentes problemas de optimización en la industria y la ingenieria (Valverde-Bourdie, 2019). Dentro de la Inteligencia Artificial (IA) en los Algoritmos Genéticos (AG), encontramos una técnica enfocada en proporcionar múltiples soluciones a problemas de optimización, los cuales nos sirven para obtener una frontera de valores entre los cuales decidir cuáles son los mejores que cumplan nuestros requerimientos. Basándonos como criterio adicional en el conocimiento y la experiencia del Tomador de Decisiones (TD), en este último campo se ha desarrollado adicionalmente una investigación para aportar y apoyar a los tomadores de decisiones, por tanto reducir al máximo la ambivalencia e imprecisión en la elección entre los valores optimizados (Maria Jose Tena Mateos et al., 2020).

Los métodos metaheurísticos juegan un papel importante en muchas áreas del conocimiento, por aquello resultan de especial interés en la resolución de problemas que no son determinísticos, sino que se convirtien en problemas de origen estocástico como se expresan en las fórmulas siguientes : 


\section{Problema Determinístico}

$\operatorname{maximizar} f(x)=\sum_{j=1}^{n} \sum_{l \in j} V_{j} \times X_{l, j}$

\section{Sujeto a:}

$\sum_{j=1}^{n} \sum_{l \in A_{j}} W_{i, j} \times X_{l, j} \leq C_{i}\left(i=1,2, \ldots, d ; l \in A_{j} ; j=1,2 \ldots, n\right)$,

$\sum_{l \in A_{j}} X_{l, j} \leq b_{j}\left(X_{l, j} \in \mathbb{Z}_{\geq 0} ; \mathrm{l} \in A_{j} ; \mathrm{j}=1,2, \ldots, \mathrm{n}\right)$.

\section{Problemas Estocástico}

$\operatorname{maximizar} f(x)=\frac{1}{\gamma} \sum_{r=1}^{\gamma} \sum_{j=1}^{n} \sum_{l \in<A_{j}} V_{j} \times S_{l, j, r}-K_{j} \times\left(X_{l, j, r}-S_{l, j, r}\right)$

$\left(r=1,2, \ldots, \gamma ; l \in A_{j} ; j=1,2, \ldots, n\right)$.

\section{Sujeto a 2 y 3}

\section{Comparado con:}

$\operatorname{maximizar} f(x)=\sum_{j=1}^{n} \sum_{l \in j} V_{j} \times X_{l, j}$

Conforme la fórmula el problema de optimización es determinístico cuando la función objetivo es lineal con variables aleatorias discretas y que puede ser resuelto con programación lineal, mientras que el problema es estocástico cuando la función deja de ser lineal, las variables aleatorias son continuas y se agrega incertidumbre, por tanto, para ser resueltos se deben usar métodos alternativos que cubran estas diferencias.

Cuando el problema es estocástico la vía de la resolución es diferente y las técnicas aplicadas son variadas, encontramos que la IA es un campo de investigación que pese a 
su antigüedad, utilizado desde 1950 (Valverde-Bourdie, 2019) y debido a la mejora en la capacidad de computo, ha ingresado con fuerza como una metaheurística de resolución de problemas de optimización (Velez \& Montoya, 2007), con un potencial extraordinario (Valverde-Bourdie, 2019). Cabe mencionar que de manera paralela surge una disciplina a partir de los resultados optimizados que entregan estos algoritmos, nos hallamos con una problemática de elegir cuáles de estos valores son los que debemos utilizar en la resolución del problema estocástico (M.J.T. Mateos et al., 2020).

El objeto de este estudio es usar IA para la optimización de procesos en la Industria Plástica, debido a que sus procesos en la mayoría de los casos resultan complejos de analizar debido a su naturaleza estocástica. Dentro de la IA el Machine Learning (ML) es un área que comprende técnicas con una diversidad de algoritmos, los cuales podemos ver clasificados en la Figura 1, cada uno de ellos han tenido un campo de aplicación dentro de la industria (Cioffi et al., 2020) y en ciertas ocasiones se aplican de manera paralela para comparar y obtener mejores resultados.

Figura 1. Clasificación de principales técnicas de Machine Learning

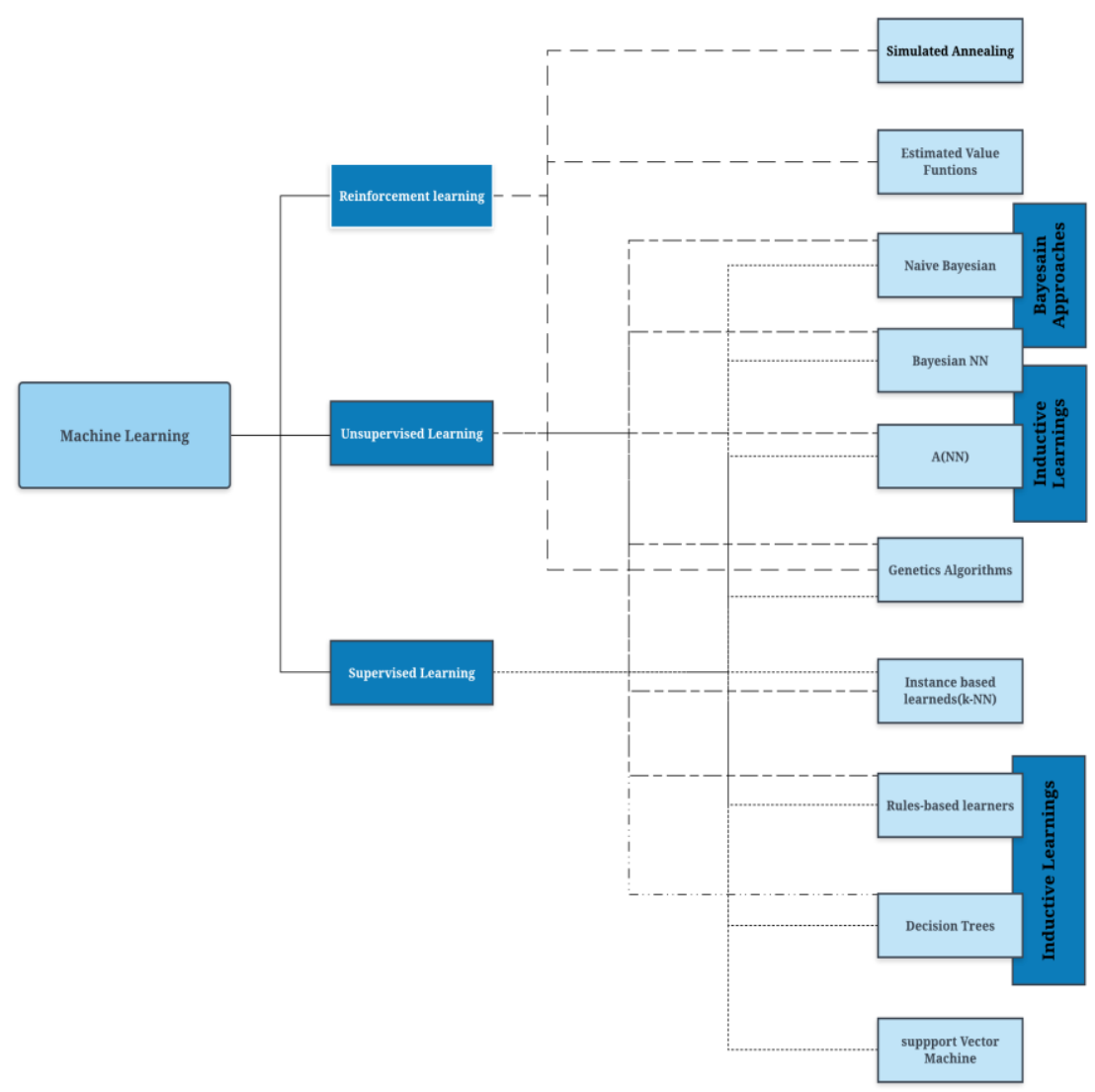

Fuente: (Wang et al., 2021) 
Los Algoritmos Genéticos resultan de especial atención en esta clasificación teniendo relación con todas las técnicas de Machine Learning, y por tanto de enorme utilidad al momento de escogerla como algoritmo de optimización y resolución de problemas en la industria.

\section{Figura 2. Algoritmos Genéticos}

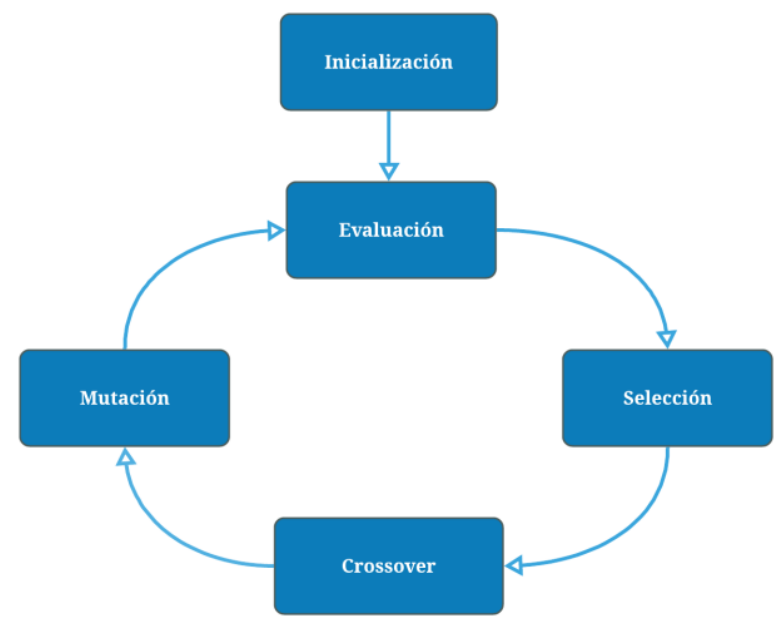

Elaboración Propia

1.1. Algoritmos Genéticos. Los AG se basan en mecanismos de evaluación, selección, crossover, mutación(Deb, 1999). Los AG son métodos iterativos y estocásticos implementados para solucionar problemas de alta complejidad (S.N.Sivanandam, 2008). Las técnicas con AG utilizadas pueden clasificarse según la Tabla 1, diferenciados en este caso por el uso del Óptimo de Pareto que son los valores resultantes de la aplicación de los algoritmos y que en conjunto se les denomina Frontera de Pareto, siendo todos los resultados optimizados posibles de un problema con objetivos múltiples y restricciones.

1.1.1. Frontera de Pareto. La frontera de pareto u óptimos de pareto contituye per se el origen de la investigación de optimización de multiples objetivos; decimos que un punto $x \in F$, es un óptimo de pareto si $\forall x \in F$

$\bigwedge_{i \in I}\left(f_{i}(\bar{x})=f_{i}(\bar{x})\right)$

$o$, hay al menos una

$\in I$ (I es el conjunto de funciones objetivo del problema) tal que

$f_{i}(\bar{x})>f_{i}(\bar{x})$ 
El óptimo de pareto expresada en la formula define el conjunto de resultados obtenidos de aplicar AG, los cuales son los mejores valores posibles en cada interación y cambio de generación.

Tabla 1. Clasificación Algoritmos Genéticos

\begin{tabular}{|c|c|c|}
\hline $\begin{array}{l}\text { Frontera } \\
\text { de Pareto }\end{array}$ & Técnica & Descripción \\
\hline NO & $\begin{array}{l}\text { VEGA (Vector Evaluated Genetic } \\
\text { Algorithm) }\end{array}$ & $\begin{array}{l}\text { Basado en selección proporcional, } \\
\text { equivalente a promediar las } \\
\text { aptitudes de cada uno de los } \\
\text { objetivos. }\end{array}$ \\
\hline NO & Ordenamiento Lexicografic & $\begin{array}{l}\text { Los objetivos se jerarquizan en un } \\
\text { orden de importancia definido por el } \\
\text { diseñador. }\end{array}$ \\
\hline NO & $\begin{array}{l}\text { Uso de Géneros para identificar } \\
\text { Objetivos }\end{array}$ & $\begin{array}{l}\text { Basado en VEGA, usando géneros } \\
\text { para definir subpoblaciones } \\
\text { independientes para cada objetivo. }\end{array}$ \\
\hline NO & Uso de Min-Max con pesos & $\begin{array}{l}\text { Basada en la teoría de juegos, Al } \\
\text { conocer los extremos de la función } \\
\text { objetivo obtenidas como problemas } \\
\text { de optimización por separado. }\end{array}$ \\
\hline SI & $\begin{array}{l}\text { Algoritmo Genético No } \\
\text { Generacional }\end{array}$ & $\begin{array}{ll}\text { La aptitud de los individuos } & \text { se } \\
\text { calcula de manera incremental es } \\
\text { decir una mejora de la } \\
\text { jerarquización ponderada. }\end{array}$ \\
\hline SI & $\begin{array}{l}\text { Uso de Pesos Generados } \\
\text { Aleatoriamente y elitismo }\end{array}$ & $\begin{array}{l}\text { Similar a mínimos a Min-Max, con } \\
\text { otra forma de generación de pesos. }\end{array}$ \\
\hline SI & Jerarquización de Pareto & $\begin{array}{l}\text { La idea básica es encontrar el } \\
\text { conjunto de cadenas de la población } \\
\text { que sean no dominadas (en el } \\
\text { sentido de Pareto). }\end{array}$ \\
\hline SI & $\begin{array}{l}\text { MOGA (Múltiple Objetive Genetic } \\
\text { Algorithm) }\end{array}$ & $\begin{array}{l}\text { Distribuir la población sobre la } \\
\text { frente de Pareto, repartiendo aptitud } \\
\text { en base a los valores de la función } \\
\text { objetivo. }\end{array}$ \\
\hline SI & $\begin{array}{l}\text { NSGA (Non-Dominated sorting } \\
\text { Genetics Algorithm) }\end{array}$ & $\begin{array}{l}\text { Basada en varias capas de } \\
\text { clasificación de los individuos, la } \\
\text { repartición de la aptitud se efectúa } \\
\text { en base a los valores de los } \\
\text { parámetros en vez de los valores de } \\
\text { la función objetivo. }\end{array}$ \\
\hline SI & NPGA (Niched Pareto GA) & $\begin{array}{l}\text { No aplica la selección de Pareto a } \\
\text { toda la población sino solo a un } \\
\text { segmento. }\end{array}$ \\
\hline
\end{tabular}

Fuente: (Coello Coello, n.d.) 


\subsection{Procesos en la manufactura del plástico}

La industria plástica en general se ha diferenciado por ser una actividad manufacturera muy dinámica, su crecimiento, desarrollo, aplicaciones e impactos en la sociedad y la economía durante los últimos años la convierte en un gran aporte en la fabricación de productos esenciales para el ser humano (Ubilla, 2019). No obstante a los aspectos negativos del uso del plástico en terminos de contaminación, derivado principalmente a la falta de un proceso de reciclaje extendido en todos los paises, esta industria aporta con productos que permiten el manejo eficiente y mejorado en el empaque de alimentos principalmente (Ubilla, 2019).

Figura 3. Principales Propiedades de los Plásticos

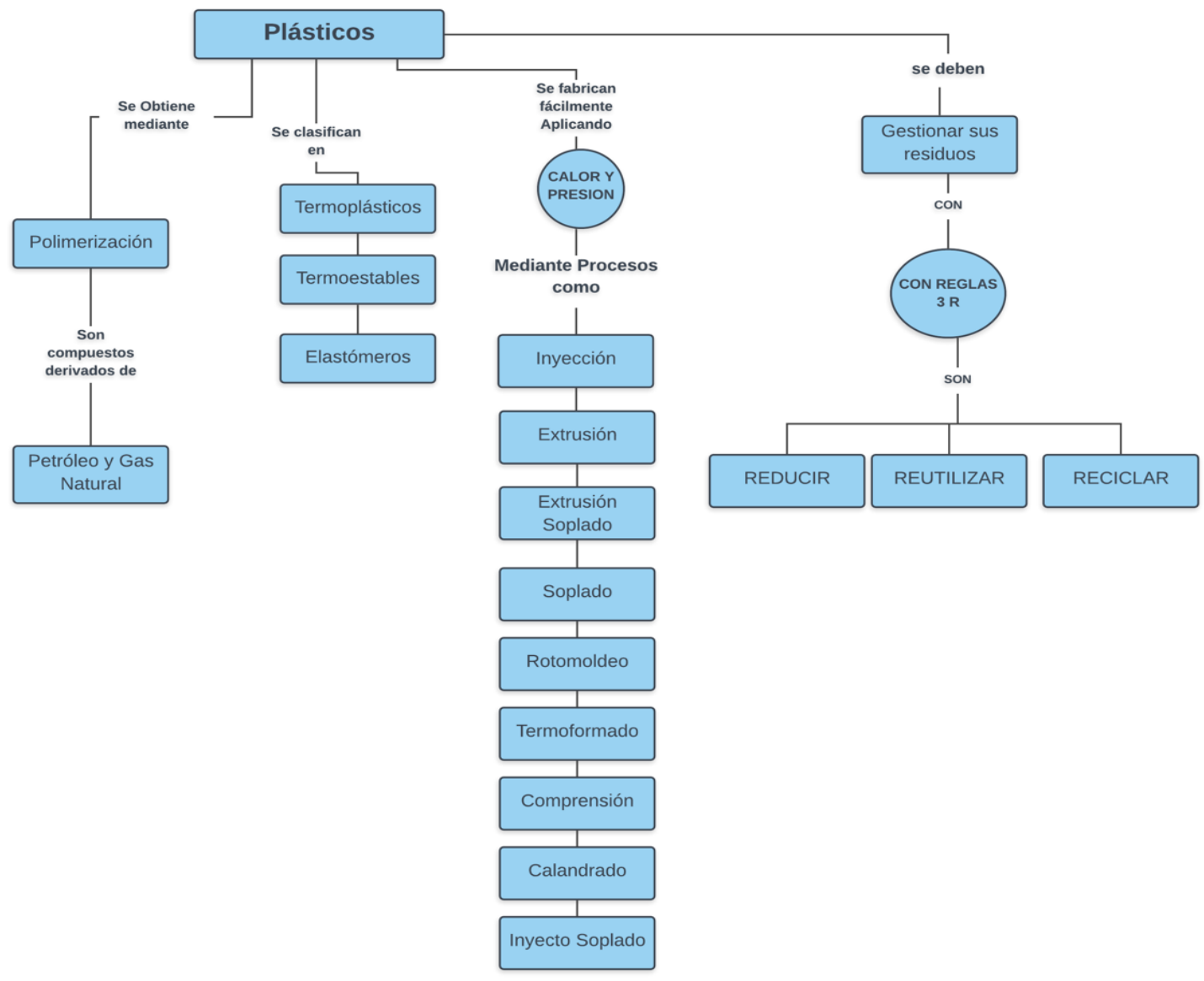

Fuente : Elaboración Propia

Como se nota en la Figura 3 y dependiendo del tipo de producto que se desea producir, los plásticos se los puede procesar mediante extrusión, soplado, extrusión soplado, termoformado, rotomoldeo, compresión, calandrado e inyecto soplado (Mamani, 2018). Diversos trabajos sobre todo orientados a la optimización de parámetros en procesos como inyección de plásticos (De los Reyes Martínez et al., 2018), problemas de 
distribución de producción en máquinas en paralelo, (Garc, 2012) y manufactura flexible (Aguilar R, 2016) especialmente usando AG, nos dan una primera visión de la gran ventaja al usar estas técnicas en la industria manufacturera del plástico.

Cada uno de estos procesos afrontan varios problemas especialmente orientado a la optimización que es la maximización y/o minimización de uno o múltiples funciones objetivo, problemas que por su complejidad, cantidad de variables y restricciones, no pueden ser resueltos por métodos tradicionales de IO, ya que no se aproximan de manera adecuada a valores óptimos ideales debido a la alta incertidumbre y ruido inherente en las variables de cada proceso.

\subsection{Análisis de la técnica de computación evolutiva}

De manera formal, podemos definir un problema de multiples objetivos y restricciones con la fórmula 1, un problema de optimización se inicia con el análisis detallado de la situación actual, a partir de cual se diseña un modelo lo más aproximado a la realidad, determinando los siguientes elementos:

1.- Funciones Objetivo

2.- Restricciones

3.- Variables

Una vez que tenemos definido el modelo, en este caso estocástico para su mejor aproximación a la realidad de un proceso de manufactura, podemos determinar el uso de AG basados en técnicas de computación evolutiva para su resolución.

Utilizamos el AG: Non-Dominated Sorting Genetic Algorithm ( NSGA2) para este modelo propuesto, debido a ser el más recomendo para problemas complejos de varios objetivos (Blank \& Deb, 2020).

El algoritmo NSGA2 fue propuesto por Srinivas y Deb (Deb et al., 2002), y se basa en el uso de varias capas de clasificación de los individuos. Antes de efectuar la selección, la población es jerarquizada en base a la no dominación: todos los individuos no dominados se clasifican en la misma categoría (con un valor arbitrario de aptitud, el cual es proporcional al tamaño de la población a fin de proporcionar un potencial reproductivo equitatativo para estos individuos. La técnica se efectúa en base a los valores de los párametros y no de los valores de la función objetivo, lo cual pretende asegurar una mejor distribución de los individuos. 


\section{METODOLOGÍA}

Para optimizar las cantidades de materiales en la producción de un producto terminado (tapa inferior amarilla), por proceso de inyección de plástico, así como definir el mejor factor de mantenimiento como recurso aleatorio del proceso debemos empezar con localizar las fuentes de información: registros control de calidad, reportes históricos de importaciones de materia prima, mantenimiento y producción-planta. Estos datos se proceden a tratarlos mediante procesos ETLs (extracción, transformación y carga), para obtener datos depurados y limpios en el período comprendido entre Enero 2018 Septiembre 2021 en las siguientes variables:

- Costo del Polietileno Alta Densidad Inyección ML 20

- Costo del Polietileno Baja Densidad Inyección ML50

- Costo del Colorante Amarillo 30

- Variación Peso Producto Terminado

Con los datos históricos depurados de estas variables procedemos a utilizar la librería en Phyton Fitter, que tienen como objetivo la lectura en fuentes de datos variados para determinar la mejor distribución de probabilidad en base al error cuadrático medio, los resultados indicados desde la Figura 4 hasta la Figura 7 nos entregan las principales distribuciones evaluadas junto con la de mayor ranking y los parámetros correspondientes.

Figura 4. Distribución de costo de MP Polietileno Alta Densidad Inyección ML 20

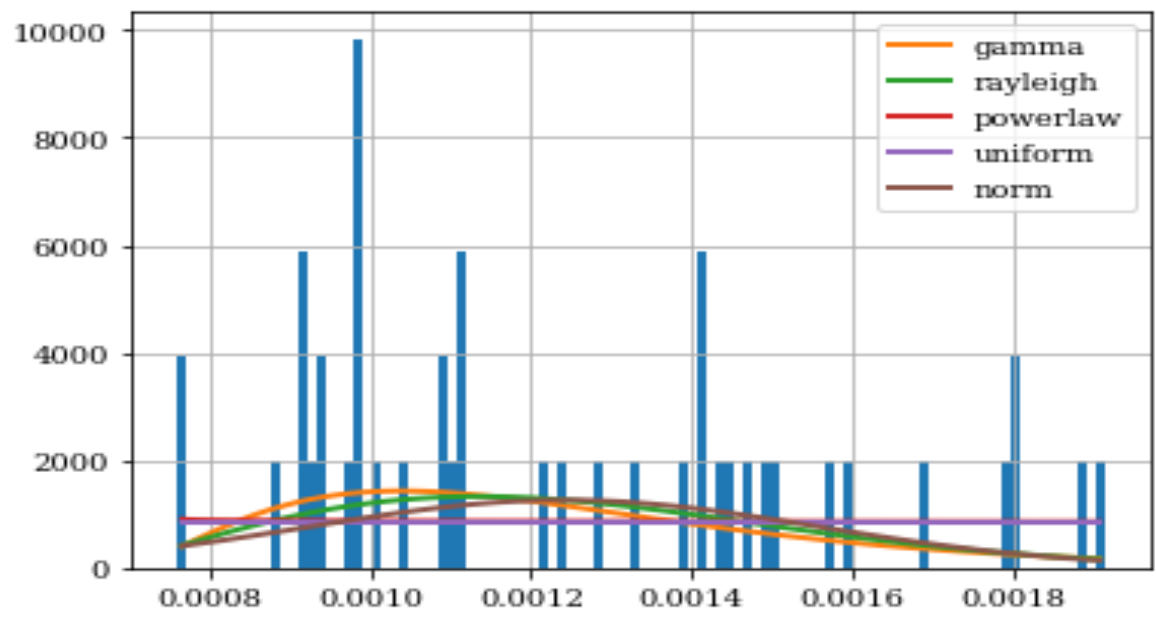

$\begin{array}{lrrrr} & \text { Sumsquare_error } & \text { aic } & \text { bic } & \text { kl_div } \\ \text { gamma } & 2.662100 \mathrm{e}+08 & -1300.413667 & 698.439243 & \text { inf } \\ \text { rayleigh } & 2.705932 \mathrm{e}+08 & -1310.542966 & 695.373622 & \text { inf } \\ \text { powerlaw } & 2.733664 \mathrm{e}+08 & -1346.759266 & 699.606463 & \text { inf } \\ \text { uniform } & 2.736876 \mathrm{e}+08 & -1348.794027 & 695.873944 & \text { inf } \\ \text { norm } & 2.768562 \mathrm{e}+08 & -1303.865269 & 696.380430 & \text { inf } \\ \text { \{'gamma ': } & (2.7206122497900553,0.0006892171500883968, & 0.00020291999279807183) \text { \} }\end{array}$


En la Figura 4 obtuvimos el resultado de las principales distribuciones de probabilidad (Gamma, Rayleig, Powerlaw, Uniforme, Normal), de los datos históricos del costo de importación del Polietileno de Alta Densidad ML 20, con lo anterior se definió la distribución gamma que es de mayor precisión en base al menor suma del error cuadrático medio.

Obtenemos la siguiente función de probabilidad gamma del costo de la materia prima Polietileno de Alta Densidad ML 20.

$$
\begin{array}{r}
f_{1}=\text { stats. gamma.rvs }(2.7206122497900553, \text { loc } \\
=0.0006892171500883968, \text { scale } \\
=0.00020291999279807183)
\end{array}
$$

Figura 5. Distribución de costo de MP Polietileno Baja Densidad Inyección ML50

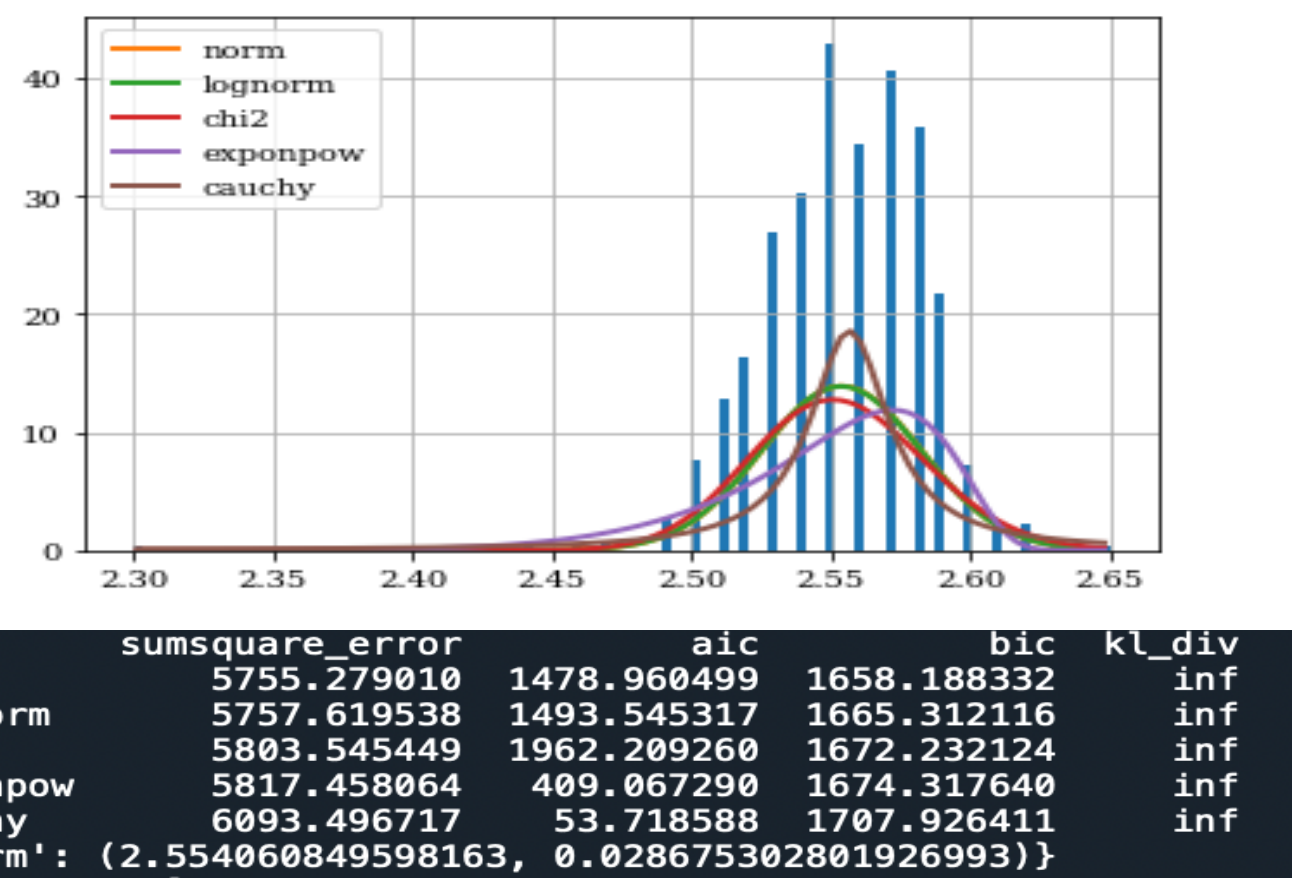

En la Figura 5 obtuvimos el resultado de las principales distribuciones de probabilidad (Normal, logNorm, Chi2, ExponPow, Cauchy), de los datos históricos del costo de importación del Polietileno de Alta Densidad ML 50, con lo anterior se definió la distribución normal que es de mayor precisión en base al menor suma del error cuadrático medio. 
Obtenemos la siguiente función de probabilidad del costo de la materia prima Polietileno de Alta Densidad ML 20.

$$
\begin{gathered}
f_{1}=\text { stats.norm. } r v s(l o c=2.554060849598163, \text { scale } \\
=0.028675302801926993)
\end{gathered}
$$

Figura 6. Distribución de Costo de Materia Prima Colorante Amarillo 30

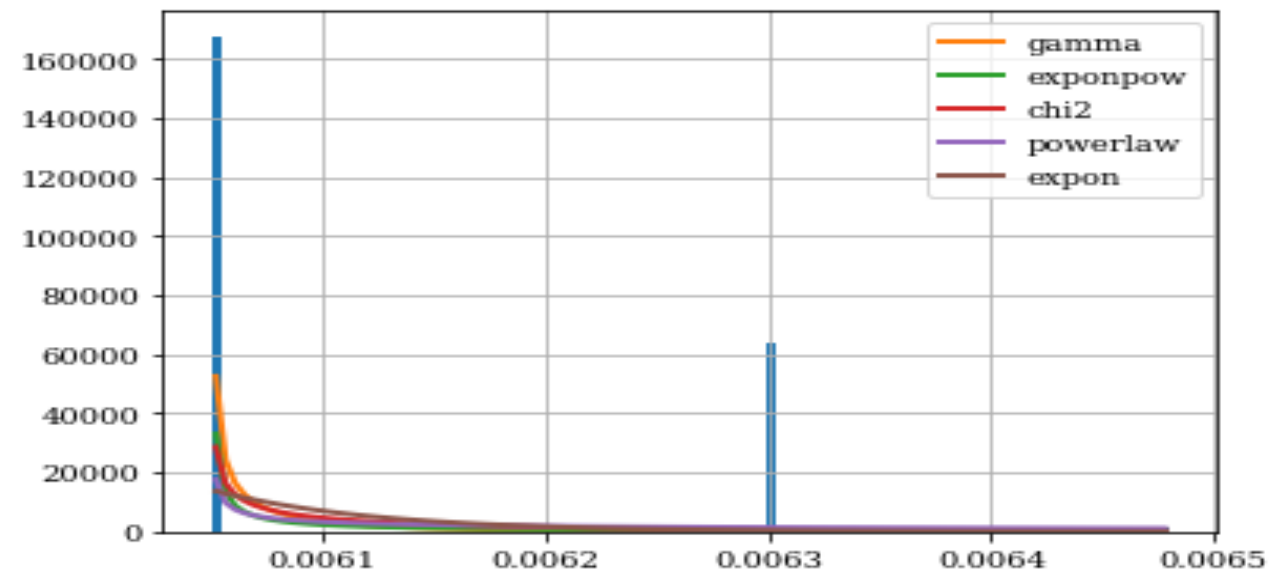

$\begin{array}{lrrrc} & \text { Sumsquare_error } & \text { aic } & \text { bic } & \text { kl_div } \\ \text { gamma } & 1.867757 \mathrm{e}+10 & -992.499607 & 3075.184008 & \text { inf } \\ \text { exponpow } & 2.259521 \mathrm{e}+10 & -1301.235765 & 3106.602426 & \text { inf } \\ \text { chi2 } & 2.439550 \mathrm{e}+10 & -1369.038927 & 3119.251466 & \text { inf } \\ \text { powerlaw } & 2.700023 \mathrm{e}+10 & -1489.653071 & 3135.990160 & \text { inf } \\ \text { expon } & 2.916336 \mathrm{e}+10 & -1299.853705 & 3143.600433 & \text { inf } \\ \text { \{'gamma': } & (0.3548583864084388,0.006049999999999999,6.860027032515168 \mathrm{e}-05)\}\end{array}$

En la Figura 6 obtuvimos el resultado de las principales distribuciones de probabilidad (Gamma, ExponPow, Chi2, PowerLaw, Expon), de los datos históricos del costo de importación del Colorante Amarillo 30, con lo anterior se definió la distribución gamma, es de mayor precisión en base a la menor suma del error cuadrático medio.

Obtenemos la siguiente función de probabilidad gamma del costo de la materia prima Polietileno de Alta Densidad ML 20.

$$
\begin{array}{r}
\mathbf{f}_{1}=\text { stats. gamma. } \operatorname{rvs}(0.3548583864084388, \text { loc } \\
=0.006049999999999999, \text { scale } \\
=6.860027032515168 \mathrm{e}-05)
\end{array}
$$


Figura 7. Distribución de Peso de Producto Terminado Tapa Amarilla

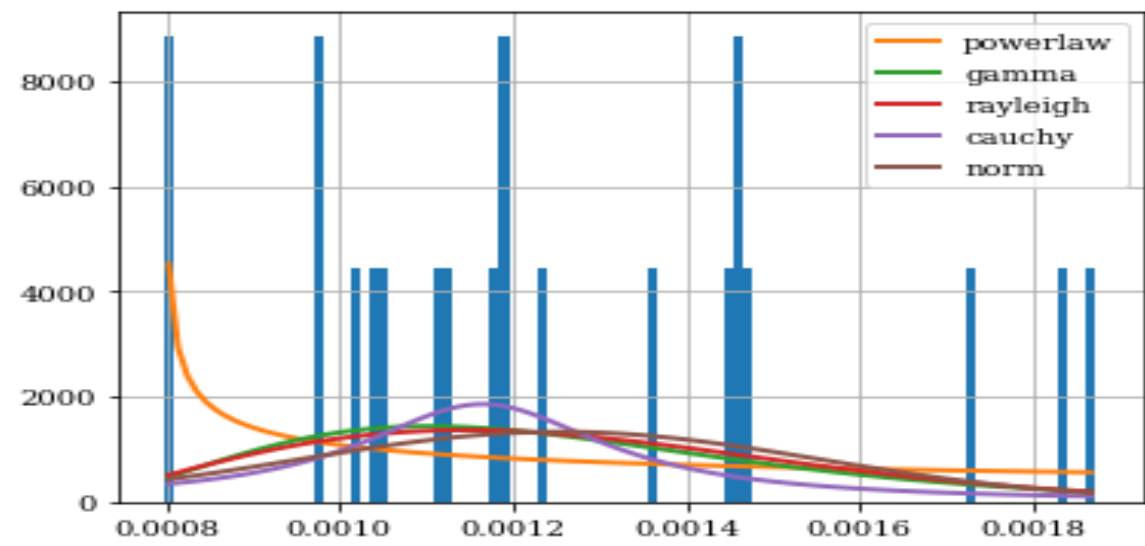

$\begin{array}{lrrrr} & \text { sumsquare_error } & \text { aic } & \text { bic } & \text { kl_div } \\ \text { powerlaw } & 4.636957 \mathrm{e}+08 & -1337.661831 & 364.248119 & \text { inf } \\ \text { gamma } & 4.770980 \mathrm{e}+08 & -1317.538147 & 364.846480 & \text { inf } \\ \text { rayleigh } & 4.780765 \mathrm{e}+08 & -1326.368807 & 361.844981 & \text { inf } \\ \text { cauchy } & 4.812183 \mathrm{e}+08 & -1245.633768 & 361.982537 & \text { inf } \\ \text { norm } & 4.829915 \mathrm{e}+08 & -1320.418252 & 362.059776 & \text { inf } \\ \{\text { 'powerlaw': } & (0.6052607224271795,0.0007975429999999998,0.001090439624933105)\} \\ {[-0.00141572]} & & \end{array}$

En la Figura 7 obtuvimos el resultado de las principales distribuciones de probabilidad (PowerLaw, Gamma, RayLeigh, Cauchi, Normal), de los datos históricos del peso de la tapa inferior amarilla, los cuales son recolectados por Control de Calidad, con lo anterior se definió la distribución norma PowerLaw, es de mayor precisión en base al menor suma del error cuadrático medio.

Obtenemos la siguiente función de probabilidad gamma, del costo de la materia prima Polietileno de Alta Densidad ML 20.

$$
\begin{aligned}
\boldsymbol{f}_{1}=\text { stats. powerlaw. } \operatorname{rvs}(0.6052607224271795, \text { loc } \\
=0.0007975429999999998, \text { scale } \\
=0.001090439624933105)
\end{aligned}
$$

Con los datos de las mejores distribuciones de probabilidad que reflejan la incertidumbre en las variables claves, procedemos a definir las funciones objetivos y restricciones para luego aplicar uno de los frameworks, más usados en optimización de múltiples objetivos PYMOO(Blank \& Deb, 2020), con uno de los AG más eficientes en desempeño NSGA2, el cual implementado en Python nos ayuda a determinar el conjunto de soluciones probables y que serán usadas por el TD, para determinar la función objetivo optimizada con la minimización del costo de producción, la disminución del factor de mantenimiento. 


\section{Las Funciones objetivos son}

- Minimizar el costo de elaboración del producto terminado.

$$
\begin{aligned}
f_{1}=\text { stats. } g a m m a . r v s(2.7206122497900553, \text { loc } \\
\\
=0.0006892171500883968, \text { scale } \\
=0.00020291999279807183) * x[0] \\
- \text { stats. powerlaw. rvs }(0.6052607224271795, \text { loc } \\
=0.0007975429999999998, \text { scale } \\
=0.001090439624933105) * x[1] \\
- \text { stats. gamma. rvs }(0.3548583864084388, \text { loc } \\
=0.006049999999999999, \text { scale } \\
=6.860027032515168 \mathrm{e}-05) * x[2]-x[3] \\
* 1.856489
\end{aligned}
$$

- Minimizar el factor costo de Mantenimiento

$f_{2}=0.0001735-x[3]$

\section{Restricciones}

- La cantidad de Polietileno Alta Densidad Inyección ML 20 es la mitad de la cantidad de Polietileno Baja Densidad Inyección ML50.

$g_{1}=0.5 * x[0]-0.5 * x[1]$

- La suma de componentes del producto no puede ser mayor a su peso, el cual tiene una distribución normal en función de los datos históricos analizados.

$$
\begin{gathered}
g_{2}=[0]+x[1]+x[2]-\text { stats. norm. } r v s(\operatorname{loc}=2.554060849598163, \text { scale } \\
=0.028675302801926993)
\end{gathered}
$$

- La cantidad de colorante es el $5.5 \%$ del peso total del producto.

$\mathrm{g}_{3}=\mathrm{x}[2]-$ stats. norm. $\mathrm{rvs}(\operatorname{loc}=2.554060849598163$, scale

$$
=\frac{0.028675302801926993}{0.0025} * 0.022
$$

Variables

- $\quad x$ [0]: Cantidad de Polietileno Alta Densidad Inyección ML 20

- $\quad x$ [1]: Cantidad de Polietileno Baja Densidad Inyección ML50

- $x$ [2]: Cantidad de Colorante Amarillo 30

- $x[3]:$ Factor de mantenimiento 


\section{RESULTADOS Y DISCUSIÓN}

Figura 9. Puntos en la Frontera de Pareto

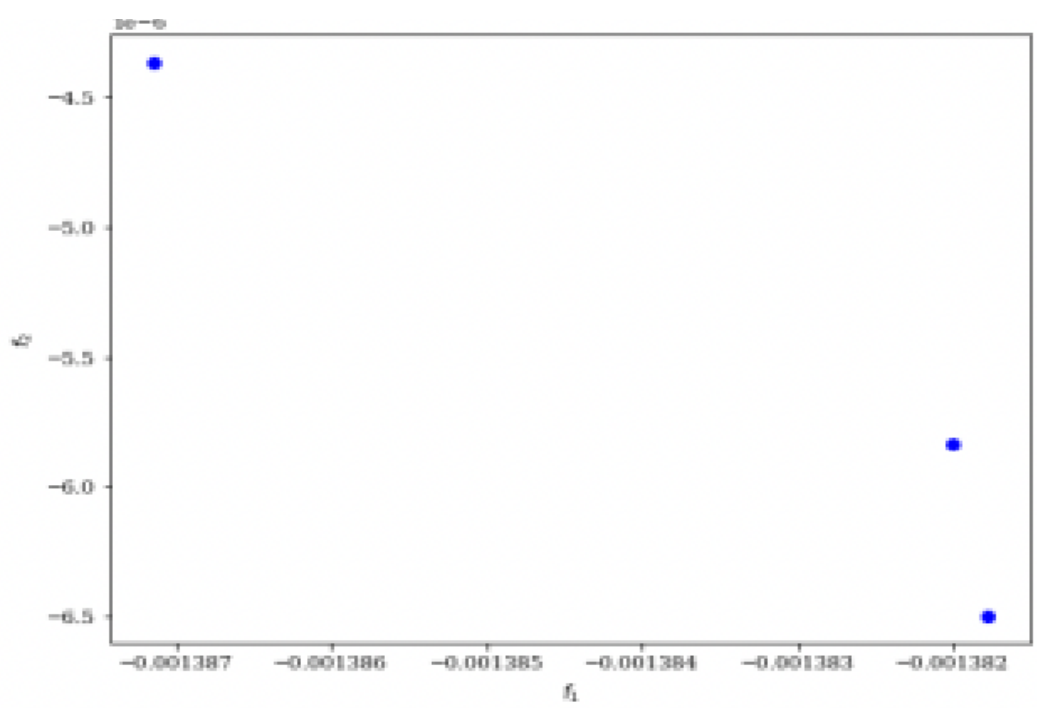

En la Figura 9 obtenemos los puntos dentro de la frontera de pareto luego de correr el algoritmo genético NSGA2 con 500 individuos de población inicial y con 500 iteraciones con mutación.

Tabla 2. Costo y Lista de Materiales Tapa Inferior Amarilla

\begin{tabular}{cccccc}
\hline $\begin{array}{c}\text { Costo } \\
\text { Producción }\end{array}$ & $\begin{array}{c}\text { Costo de } \\
\text { Mantenimiento }\end{array}$ & $\begin{array}{c}\text { Cantidad } \\
\text { MP1 }\end{array}$ & $\begin{array}{c}\text { Cantidad } \\
\text { MP2 }\end{array}$ & $\begin{array}{c}\text { Cantidad } \\
\text { MP3 }\end{array}$ & $\begin{array}{c}\text { Factor } \\
\text { Manteniento }\end{array}$ \\
\hline 0.005472 & 0.000462 & 1.30000000 & 1.30000000 & 0.02600000 & 0.17350000
\end{tabular}

Tabla 3. Costo y Lista de Materiales Optimizado Tapa Inferior Amarilla

\begin{tabular}{cccccc}
\hline $\begin{array}{c}\text { Costo } \\
\text { Producción }\end{array}$ & $\begin{array}{c}\text { Costo de } \\
\text { Mantenimiento }\end{array}$ & $\begin{array}{c}\text { Cantidad } \\
\text { MP1 }\end{array}$ & $\begin{array}{c}\text { Cantidad } \\
\text { MP2 }\end{array}$ & $\begin{array}{c}\text { Cantidad } \\
\text { MP3 }\end{array}$ & $\begin{array}{c}\text { Factor } \\
\text { Mantenimiento }\end{array}$ \\
\hline 0.00138201 & 0.00000583 & 1.208157 & 1.203429 & 0.021239 & 0.000179 \\
0.00138715 & 0.00000436 & 1.238100 & 1.225436 & 0.020852 & 0.000178 \\
0.00138177 & 0.00000650 & 1.208157 & 1.203429 & 0.022145 & 0.000180
\end{tabular}

En la Tabla 3 podemos observar el detalle de los tres puntos de la Frontera de Pareto de la Figura 9, las dos funciones por minimizar: Costo de Producción y Costo de mantenimiento junto con el valor de las variables del modelo.

El procedimiento aplicado resulta sumamente eficiente en este problema de optimización múltiple, obteniendo valores ideales en la lista de materiales y el factor de mantenimiento y por tanto una disminución en el costo de producción. 
Los resultados obtenidos según la Tabla 3, representan una disminución del costo de producción de un $75 \%$ con respecto a las cantidades usadas actualmente de manera estándar y de manera empírica según la Tabla 2.

En la Figura 9, podemos observar la frontera generada de valores optimizados, de los cuales podemos escoger cual de ellos utilizar en nuestra lista de materiales y así obtener el costo optimizado junto con el valor de mantenimiento sugerido para alcanzar los dos objetivos de manera simultánea.

\section{CONCLUSIONES}

Con la aplicación del AG: NSGA2 usando el framework Pymoo basado en Phyton, obtuvimos un valor de función objetivo minimizando el costo de producción de la tapa amarilla en un $75 \%$ con respecto al costo actual, el factor de mantenimiento, así como el valor optimizado de sus materias primas principales, con esto se pueden ajustar las listas de materiales que se usan actualmente para una mejor aproximación del consumo de estas y por ende una optimización en el proceso y una disminución de ajustes en el inventario final.

El uso de AG en la optimización de procesos de la industria y específicamente en la plástica tiene un campo de acción extensa, pasando por la determinación de parámetros de máquina para optimizar la producción, minimizar la materia prima y recursos para producir un producto, la planificación de la producción, mantenimiento predictivo.

A partir de esta investigación se pueden ir generando modelos en cada problemática usando AG, así como mejorarlos con el uso de algoritmos de Machine Learning y Deep Learning que son áreas de la IA actualmente de gran aplicación.

\section{REFERENCIAS BIBLIOGRÁFICAS}

Aguilar R, E. (2016). Algoritmo genético aplicado a sistemas de manufactura flexible.

Blank, J., \& Deb, K. (2020). Pymoo: Multi-Objective Optimization in Python. IEEE Access, 8, 89497-89509. https://doi.org/10.1109/ACCESS.2020.2990567

Cioffi, R., Travaglioni, M., Piscitelli, G., Petrillo, A., \& De Felice, F. (2020). Artificial intelligence and machine learning applications in smart production: Progress, trends, and directions. Sustainability (Switzerland), 12(2). https://doi.org/10.3390/su12020492

Coello Coello, C. A. (n.d.). Optimización Evolutiva con Objetivos Múltiples : Estado del Arte y Tendencias Futuras. 
De los Reyes Martínez, T. I., Aguayo, L. F. D., Ramírez, S. J. G., Gutiérrez, J. Z., \& Martínez, A. B. E. (2018). Aplicación de técnicas computo evolutivas para la optimización del proceso de inyección de plástico. Jóvenes En La Ciencia, 3(1), 483-488. http://rinfi.fi.mdp.edu.ar/handle/123456789/358

Deb, K. (1999). Introduction to genetic algorithms. Sadhana - Academy Proceedings in Engineering Sciences, 24(4), 293-315. https://doi.org/10.1007/BF02823145

Deb, K., Pratap, A., Agarwal, S., \& Meyarivan, T. (2002). A fast and elitist multiobjective genetic algorithm: NSGA-II. IEEE Transactions on Evolutionary Computation, 6(2), 182-197. https://doi.org/10.1109/4235.996017

Garc, I. D. (2012). Optimización de la producción en máquinas en paralelo de inyección de plástico. 103. http://cd.dgb.uanl.mx/bitstream/handle/201504211/5231/20446.pdf?sequence=1

Hillier, F. S., \& Lieberman, G. J. (2013). Introducción a la Investigación de operaciones. In Journal of Chemical Information and Modeling (Vol. 53, Issue 9).

Mamani, L. F. (2018). Optimización del Proceso Productivo en el Área de Producción de una Industria Plástica. Universidad Peruana de Ciencias Aplicadas (UPC), 354. http://hdl.handle.net/10757/624502

Mateos, M.J.T., Lopez-Pujalte, C., \& Canavate, A. M. (2020). Decision Support System based on Competitive Intelligence and Genetic Algoritms | Sistema de apoyo a decisiones basado en Inteligencia Competitiva y Algoritmos Genéticos. Iberian Conference on Information Systems and Technologies, CISTI, 2020-June. https://doi.org/10.23919/CISTI49556.2020.9141060

Mateos, Maria Jose Tena, Lopez-Pujalte, C., \& Canavate, A. M. (2020). Decision Support System based on Competitive Intelligence and Genetic Algoritms. Iberian Conference on Information Systems and Technologies, CISTI, 2020-June(June). https://doi.org/10.23919/CISTI49556.2020.9141060

S.N.Sivanandam, S. . D. (2008). Sivanandam_Introduction to Genetic Algorithms (Springer-Verlag (ed.)).

Ubilla, Y. (2019). "La evolución de la industria plástica en el Ecuador." http://repositorio.ug.edu.ec/bitstream/redug/41415/1/T-UBILLA ROJAS YANINA JESSICA.pdf

Valverde-Bourdie, S. (2019). Aplicaciones De La Inteligencia Artificial En La Empresa. 
https://repositorio.unican.es/xmlui/bitstream/handle/10902/17521/VALVERDE BOURDIESANDRA.pdf? sequence $=1 \&$ isAllowed $=\mathrm{y}$

Velez, M., \& Montoya, J. (2007). Solución De Problemas Combinatorios En Administración De Operaciones. Revista EIA, 8(1), 99-115. http://www.scielo.org.co/scielo.php?script=sci_arttext\&pid=S179412372007000200009

Wang, Q., Jiao, W., Wang, P., \& Zhang, Y. M. (2021). A tutorial on deep learning-based data analytics in manufacturing through a welding case study. Journal of Manufacturing Processes, 63(December 2019), 2-13. https://doi.org/10.1016/j.jmapro.2020.04.044 